\title{
AJUSTE DE MODELOS SEMITEÓRICOS PARA PREDIÇÃo DE CINÉTICA DE SECAGEM DE YACON (Smallanthus sonchifolius)
}

\author{
F. J. LOPES ${ }^{1 *}$, R. E. MELLO JR ${ }^{1}$, G. E. ALVES ${ }^{1}$, K. S. MENDONÇA ${ }^{1}$, K.C.R. \\ SILVA $^{1}$, J. L. G. CORREA ${ }^{1}$ \\ ${ }^{1}$ Universidade Federal de Lavras, Departamento de Ciências dos Alimentos \\ *e-mail:francemirlopes@yahoo.com.br
}

\begin{abstract}
RESUMO
O presente trabalho teve como objetivo estudar a cinética de secagem convectiva de yacon, bem como testar o ajuste com diferentes modelos matemáticos. Os experimentos foram realizados nas temperaturas de $40 ; 50 ; 60$ e $70^{\circ} \mathrm{C}$ e velocidade do ar constante de $0,5 \mathrm{~m} / \mathrm{s}$. A secagem do yacon ocorreu em taxa decrescente com maiores taxas de redução do teor de umidade no início da secagem. Os menores tempos de secagem foram observados com o aumento da temperatura. Os modelos matemáticos de Midilli e colaboradores, Page, Henderson e Pabis e de Doymaz apresentaram ótimos ajustes aos dados experimentais com $\mathrm{R}^{2}$ maiores que 0,99 e $\chi^{2}$ menores que $2,18 \times 10^{-5}$. Dentre estes, ainda se destacou o modelo de Midilli e colaboradores com menores valores de RMSE e Chi-quadrado que os demais.
\end{abstract}

\section{INTRODUÇÃO}

O yacon (Smallanthus sonchifolius) é originário dos vales andinos da Colômbia, Equador, Peru, Bolívia e noroeste da Argentina, em altitudes de 2.000 a 3.100 metros. Nessa região, é cultivado desde a antiga civilização Inca, e utilizado na alimentação humana (VILHENA; CÂMARA; KAKIHARA, 2000).

No Brasil, a espécie foi introduzida por volta de 1989, na região de Capão Bonito (SP), por imigrantes japoneses, que utilizam suas folhas e raízes tuberosas nos tratamentos contra diabetes e altas taxas de colesterol no sangue (KAKIHARA et al., 1996). Esta espécie vem despertando o interesse dos pesquisadores devido ao seu potencial como alimento funcional (CASTRO et al., 2013; CHOQUE DELGADO et al., 2013; VAZTOSTES et al., 2014). Diferente da maioria das raízes que armazenam carboidratos na forma de amido, o yacon armazena os carboidratos na forma de frutanos. Porém, com o armazenamento ocorre hidrólise dos frutanos (GRAEFE et al., 2004).

A conservação pela secagem se baseia no fato de que tanto os microrganismos como as enzimas e todo o mecanismo metabólico, necessitam de certa quantidade de água para suas atividades. Com a redução da água disponível, serão reduzidas a atividade de água e a velocidade das reações químicas no produto, bem como o desenvolvimento de microrganismos (DARVISHI et al., 2014).

Estudos de secagem de alimentos têm sido realizados no desenvolvimento de modelos semiteóricos, já que os modelos empíricos omitem os fundamentos do processo de secagem e seus parâmetros não têm significado físico. Tais modelos semiteóricos concorrem para que haja 


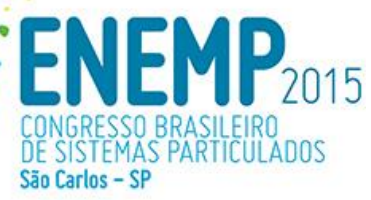

harmonia entre a teoria e a facilidade de uso. Entre os modelos semiteóricos, o modelo de Page, Henderson e Pabis, Logarítmica e o de Midilli e colaboradores, têm sido amplamente utilizados (MENEZES et al., 2013).

O presente trabalho teve como objetivo estudar a cinética de secagem convectiva de yacon em diferentes temperaturas do ar de secagem e testar o ajuste de modelos empíricos aos dados experimentais.

\section{MATERIAL E METODOS}

\subsection{Material}

Tubérculos de yacon (Smallanthus sonchifolius) foram obtidos junto ao comércio local de Lavras, Minas Gerais. Para seleção da matéria-prima foram considerados o grau de maturação, tamanho, peso, intensidade de cor e firmeza uniformes. Além disso, a ausência de doenças, fissuras e outras injúrias físicas foram levadas em consideração.

Os tubérculos foram utilizados nos experimentos logo após sua aquisição.

\subsection{Preparo das amostras}

As raízes selecionadas foram lavadas em água corrente. Os tubérculos foram descascados manualmente e cortados com auxilio de um molde e uma faca de aço inox nas dimensões de 2,00 x 2,00 x 0,50 cm (comprimento $\mathrm{x}$ largura $\mathrm{x}$ espessura). As dimensões das fatias foram aferidas com um paquímetro digital (Western, 150mm-DC-60, China).

\subsection{Secagem}

A secagem das amostras foi realizada em um secador de camada fixa (Conscientec, Belo Horizonte, Brasil) (Figura 1) que permite o controle do fluxo e da temperatura do ar de secagem por meio de um sistema de automação. Âs temperaturas utilizadas no processo de secagem foram de 40;50; 60 e $70^{\circ} \mathrm{C}$ e velocidade do ar mantida constante em $0,5 \mathrm{~m} \mathrm{~s}^{-1}$.
A massa das amostras foi determinada em balança semi-analítica (Shimatzu, AUY220, Kyoto, Japão) com precisão 0,1 mg a cada 10 minutos de secagem nos primeiros 60 minutos e a cada 30 minutos no restante do processo, até peso constante.

Figura 1. Secador de camada fixa utilizado na secagem

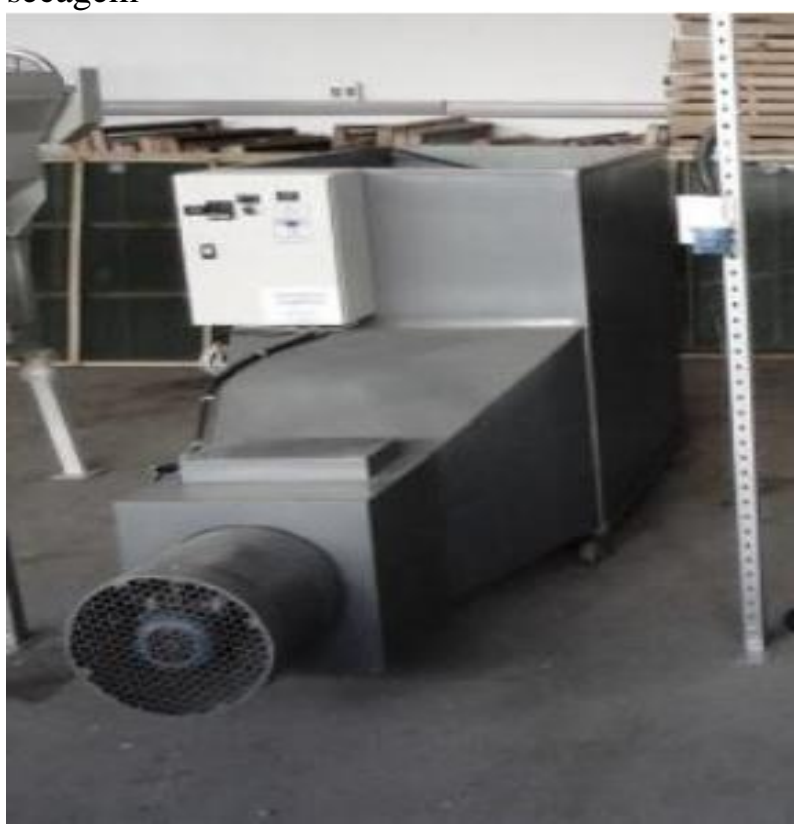

\subsection{Análises}

2.4.1 Determinação do teor de umidade

$O$ teor de umidade foi determinado segundo método gravimétrico 934.06 da AOAC (2005) em estufa a vácuo (pressão $\leq$ $100 \mathrm{mmHg}$ ) a $70^{\circ} \mathrm{C}$ até peso constante.

\subsubsection{Atividade de água}

A determinação da atividade de água foi realizada à temperatura de $25^{\circ} \mathrm{C}$ com o uso de um medidor de atividade de água (Aqualab Decagon Devices Inc. Pullman, modelo CX2T, Washington, EUA).

\subsection{Cinética de secagem}

A cinética de secagem foi obtida experimentalmente com a determinação de valores de teor de umidade com o tempo e 
modelada matematicamente com os modelos

apresentados na Tabela 1, Equação 1 a 4.

Tabela 1 - Modelos matemáticos usados para ajustes da cinética de secagem

\begin{tabular}{lll}
\hline Modelo & Equação & Referência \\
\hline Page & $M_{r}=e^{-k t^{n}}$ & PAGE (1949) \\
Henderson e Pabis & $M_{r}=a e^{(-k t)}$ & HENDERSON e PABIS (1961) \\
Logarítmica & $M_{r}=a e^{(-k t)}+c$ & DOYMAZ (2008) \\
Midilli et al. & $M_{r}=a e^{\left(k t^{n}\right)}+b t$ & MIDILLI; KUCUK; YAPAR (2002)
\end{tabular}

a,b,c, k e n são parâmetros de ajuste.

A umidade adimensional (MR) foi definida como:

$M R=\frac{\left(M_{t}-M_{e}\right)}{\left(M_{0}-M_{e}\right)}$

onde $\mathrm{M}_{\mathrm{t}}$ é o teor de umidade do yacon em cada momento, $\mathrm{M}_{0}$ teor de umidade inicial do yacon e $\mathrm{M}_{\mathrm{e}}$ teor umidade de equilíbrio.

Para avaliar o ajuste dos modelos, foi usado o coeficiente de determinação $\left(\mathrm{R}^{2}\right)$ (Equação 6), raiz do erro quadrático médio (RMSE) (Equação 7) e o valor de chiquadrado $(\chi 2)$ (Equação 8 ):

$$
\begin{aligned}
& R^{2}=\frac{\sum_{i=1}^{n}\left(M R_{\text {pred }, i}-\overline{M R}\right)^{2}}{\sum_{i=1}^{n}\left(M R_{\text {exp }, i}-\overline{M R}\right)^{2}} \\
& R S M E=\left[\frac{1}{n} \sum_{i=1}^{n}\left(M R_{\text {exp } i}-M R_{\text {pred }, i}\right)^{2}\right]^{1 / 2} \\
& \chi^{2}=\frac{\sum_{i=1}^{n}\left(M R_{\text {exp }, i}-M R_{\text {pred }, i}\right)^{2}}{N-n}
\end{aligned}
$$

Onde $\mathrm{MR}_{\text {exp,i }}$ e $\mathrm{MR}_{\text {prev,i }}$ são respectivamente os valores experimentais e previstos da razão de umidade para a observação i, $\mathrm{N}$ é o número de observações e n, o número de parâmetros no modelo.

\section{RESULTADOS E DISCUSSÃO}

O teor umidade do yacon in natura é de $8,58 \pm 0,37 \mathrm{~kg}$ de água/kg matéria seca e atividade de água $\left(\mathrm{a}_{\mathrm{w}}\right)$ de $0,990 \pm 0,001$. A secagem proporcionou a redução do teor de umidade e da atividade de água, como esperado. A atividade de água final no equilíbrio resultou em valores menores que $0,417 \pm 0,001$.

$\mathrm{Na}$ Figura 2, é apresentada a cinética de secagem nas temperaturas de 40,50, 60 e $70^{\circ} \mathrm{C}$, bem como o ajuste do modelo de Midilli et al.

Figura 2. Cinética de secagem do yacon

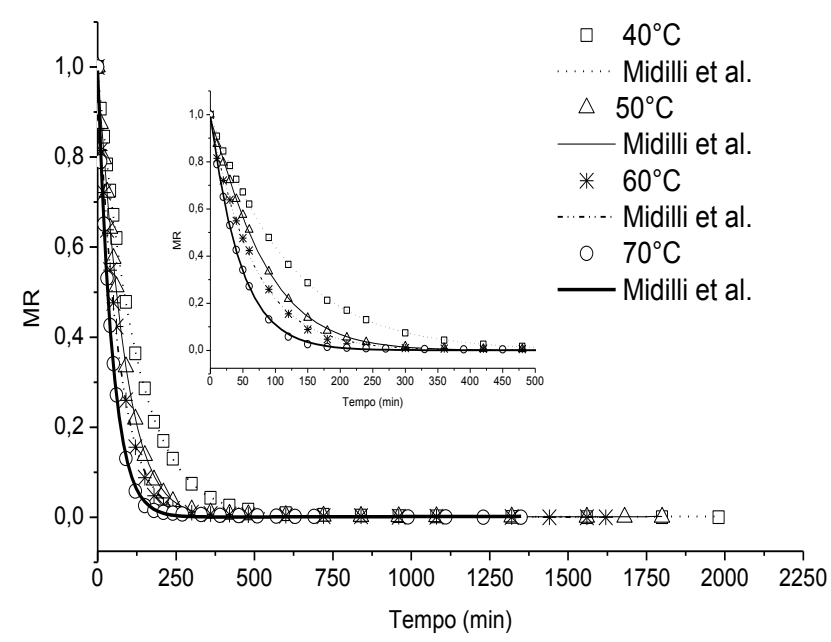


Observou-se a secagem de yacon ocorreu com taxa decrescente, indicando uma maior resistência à transferência de calor e massa do interior do produto para sua superfície do que da superfície para o ambiente e, portanto, a difusão é o principal mecanismo de transporte de umidade. As maiores taxas de redução do teor de umidade ocorreram no início da secagem. No final da secagem a umidade encontra-se fortemente ligada necessitando de maior energia para sua evaporação, resultando em menores valores da taxa de redução do teor de umidade. Além disso, menores tempos de secagem foram observados com o aumento da temperatura. $\mathrm{O}$ aumento da temperatura resulta em maior excitação das moléculas de água no interior do material, com consequente aumento da taxa de secagem nestas condições. Esses resultados estão de acordo com Purkayastha et al. (2013), Vega-Gálvez et al. (2012), Djendoubi Mrad et al. (2012) na secagem de convectiva de tomate, maça e pêra.

Segundo CASTILLO ALFARO e VIDAL MELGAREJO (2009) os principais fatores limitantes para a utilização de temperaturas elevadas no processo de secagem de yacon é a despolimerização dos frutooligossacarídeos (FOS). Segundo SCHER et al. (2009), a secagem deste produto deve ser realizada em temperaturas de até $70^{\circ} \mathrm{C}$.

Na Tabela 2 estão apresentados os resultados dos ajustes dos modelos aos dados experimentais (coeficiente de determinação $\left(\mathrm{R}^{2}\right)$, valor do chi-quadrado $\left(\chi^{2}\right)$ e raiz do erro quadrático médio (RSME).

Tabela 2 - Parâmetros de ajuste dos modelos da cinética de secagem do yacon, coeficiente de determinação $\left(\mathrm{R}^{2}\right)$.

\begin{tabular}{|c|c|c|c|c|c|c|c|c|c|}
\hline Modelo & $\mathrm{T}\left[{ }^{\circ} \mathrm{C}\right]$ & $\mathrm{R}^{2}$ & $\chi^{2} \times 10^{5}$ & RSME & $\mathrm{k}$ & $\mathrm{n}$ & $\mathrm{a}$ & $\begin{array}{c}\mathrm{b} \\
\times 10^{7}\end{array}$ & $\mathrm{c}$ \\
\hline \multirow{4}{*}{ Page } & 40 & 0,9997 & 2,59 & 0,0051 & 0,0073 & 1,026 & - & - & - \\
\hline & 50 & 0,9992 & 8,68 & 0,0093 & 0,0077 & 1,1026 & - & - & - \\
\hline & 60 & 0,9983 & 15,4 & 0,0124 & 0,0159 & 0,9902 & - & - & - \\
\hline & 70 & 0,9995 & 4,09 & 0,0064 & 0,0202 & 1,0197 & - & - & - \\
\hline \multirow{4}{*}{$\begin{array}{c}\text { Handerson e } \\
\text { Pabis }\end{array}$} & 40 & 0,9996 & 3,94 & 0,0062 & 0,0083 & - & 1,0031 & - & - \\
\hline & 50 & 0,9978 & 22,9 & 0,0151 & 0,0123 & - & 1,0176 & - & - \\
\hline & 60 & 0,9985 & 13,6 & 0,0116 & 0,0149 & - & 0,9849 & - & - \\
\hline & 70 & 0,9994 & 4,57 & 0,0067 & 0,0217 & - & 0,9993 & - & - \\
\hline \multirow{4}{*}{ Logarítmica } & 40 & 0,9996 & 4,07 & 0,0063 & 0,0083 & - & 1,0037 & - & $-0,00091$ \\
\hline & 50 & 0,9978 & 2,29 & 0,0151 & 0,0122 & - & 1,0204 & - & $-0,00408$ \\
\hline & 60 & 0,9984 & 14,1 & 0,0118 & 0,0149 & - & 0,9857 & - & $-0,00128$ \\
\hline & 70 & 0,9994 & 4,78 & 0,0069 & 0,0217 & - & 0,9991 & - & 0,00027 \\
\hline \multirow{4}{*}{ Midilli et al. } & 40 & 0,9998 & 2,18 & 0,0046 & $-0,0066$ & 1,044 & 0,9904 & 8,08 & - \\
\hline & 50 & 0,9993 & 7,19 & 0,0084 & $-0,0064$ & 1,1379 & 0,9813 & 8,25 & - \\
\hline & 60 & 0,9984 & 14,2 & 0,0119 & $-0,0135$ & 1,0214 & 0,9791 & 4,97 & - \\
\hline & 70 & 0,9995 & 4,07 & 0,0063 & $-0,0192$ & 1,0305 & 0,9925 & 1,53 & - \\
\hline
\end{tabular}

Em termos do coeficiente de determinação, todos os modelos ajustados representam bem a cinética de secagem do tubérculo yacon. Em todos os casos $\mathrm{R}^{2}>0,99$. Resultados semelhantes foram relatados por Zhu et al. (2014), Shi et al. (2013), Joykumar
Singh et al. (2012) na secagem convectiva de pêssego, yacon e batata doce, respectivamente.

Além disso, os resultados indicaram que os menores valores de RMSE e Chi-quadrado foram obtidos no modelo de Midilli e 
colaboradores, mostrando, assim, o seu elevado potencial de representação dos dados experimentais em todas as condições de secagem estudadas. Shi et al. (2013) também observaram que o modelo de Midilli et al. como sendo o modelo mais adequado para descrever o comportamento de secagem de fatias de yacon.

Analisando-se o comportamento do modelo de Page, observa-se que o parâmetro $\mathrm{k}$, que representa a constante da taxa de secagem aumentou com o aumento da temperatura, o mesmo comportamento de $\mathrm{k}$ foi observado nos modelos de Henderson e Pabis, Logarítmica. Estes resultados estão de acordo com Janjai et al. (2011), Guiné et al. (2011). O parâmetro $\mathrm{n}$ foi menor na temperatura de $60^{\circ} \mathrm{C}$ nos modelos de Page e Midilli et al. e maiores em temperaturas de $50^{\circ} \mathrm{C}$.

O parâmetro a dos modelos de Henderson e Pabis e Logarítmica diminuíram com o aumento da temperatura. No modelo de Midilli et al. observa-se que o parâmetro $b$ aumentou com a elevação da temperatura.

\section{CONCLUSÃO}

A secagem de yacon ocorreu em taxa decrescente. As maiores taxas de redução do teor de umidade ocorreram no início da secagem.

Menores tempos de secagem foram observados com o aumento da temperatura.

Dentre os modelos matemáticos Midilli e colaboradores foi o que melhor se ajustou aos dados experimentais de cinética de secagem.

Os parâmetros do modelo corroboram com aumento da taxa de secagem.

\section{NOMENCLATURA}

MR Razão de umidade, adimensional $\mathrm{T} \quad$ Temperatura $\left[{ }^{\circ} \mathrm{C}\right]$

$\begin{array}{ll}\mathrm{t} & \text { Tempo [min] } \\ \text { Subscritos } & \\ 0 & \text { Condição inicial } \\ \mathrm{f} & \text { Condição final } \\ \mathrm{eq} & \text { Equilíbrio }\end{array}$

\section{REFERÊNCIAS}

AOAC. Official methods of analysis of Association of official Analytical Chemists International.: Gainsthersburg: Horwitz 2005.

CASTILLO ALFARO, M. E.; VIDAL MELGAREJO, S. A. L. El Yacón: una nueva alternativa en la prevención y tratamiento de la salud. Santa Fe, Argentina: El Cid Editor apuntes, 2009.

CASTRO, A.; CÉSPEDES, G.; CARBALLO, S.; BERGENSTÅHL，B.; TORNBERG，E. Dietary fiber, fructooligosaccharides, and physicochemical properties of homogenized aqueous suspensions of yacon (Smallanthus sonchifolius). Food Research International, v.50, n.1, p.392-400, 2013.

CHOQUE DELGADO, G.; DA SILVA CUNHA TAMASHIRO, W.; MARÓSTICA JUNIOR, M.; PASTORE, G. Yacon (Smallanthus sonchifolius): A Functional Food. Plant Foods for Human Nutrition, v.68, n.3, p.222-228, 2013.

DARVISHI, H.; ASL, A. R.; ASGHARI, A.; AZADBAKHT, M.; NAJAFI, G.; KHODAEI, J. Study of the drying kinetics of pepper. Journal of the Saudi Society of Agricultural Sciences, v.13, n2, p.130-138, 2014

DJENDOUBI MRAD, N., BOUDHRIOUA, N., KECHAOU, N., COURTOIS, F., \& BONAZZI, C. Influence of air drying temperature on kinetics, physicochemical 
properties, total phenolic content and ascorbic acid of pears. Food and Bioproducts Processing, v. 90, n. 3, p. 433-441, 2012.

DOYMAZ, I. Convective drying kinetics of strawberry. Chemical Engineering and Processing: Process Intensification, v. 47, n. 5, p. 914-919, 2008.

GRAEFE, S.; HERMANN, M.; MANRIQUE, I.; GOLOMBEK, S.; BUERKERT, A. Effects of post-harvest treatments on the carbohydrate composition of yacon roots in the Peruvian Andes. Field Crops Research, v.86, n.23, p.157-165, 2004.

GUINÉ, R. P. F.; PINHO, S.; BARROCA, M. J. Study of the convective drying of pumpkin (Cucurbita maxima). Food and Bioproducts Processing, v.89, n.4, p.422-428, 2011.

HENDERSON, S.M.; PABIS, S. Grain drying theory II: Temperature effects on drying coefficients. Journal of Agricultural Engineering Research, n.6, p.169-174, 1961.

JANJAI, S.; PRECOPPE, M.; LAMLERT, N.; MAHAYOTHEE, B.; BALA, B. K.; NAGLE, M.; MÜLLER, J. Thin-layer drying of litchi (Litchi chinensis Sonn.). Food and Bioproducts Processing, v.89, n.3, p.194201, 2011.

JOYKUMAR SINGH, N.; PANDEY, R. K. Convective air drying characteristics of sweet potato cube (Ipomoea batatas L.). Food and Bioproducts Processing, v.90, n.2, p.317322, 2012.

KAKIHARA, T. S.; CÂMARA, F. L. A.; VILHENA, S. M. C.; RIERA, L. Cultivo e industrialização de yacon (Polymnia sonchifolia): uma experiência brasileira. In: Congresso latino americano de raízes tropicais 1 e congresso brasileiro de mandioca 9, São Pedro. p.148, 1996.
MENEZES, M. L.; KUNZ, C. C.; PERINE, P.; PEREIRA, N. C.; DOS SANTOS, O. A. A., DE BARROS, S. T. D. Analysis of convective drying kinetics of yellow passion fruit bagasse. Acta Scientiarum-Technology, v. 35, n. 2, p. 291-298, 2013.

MIDILLI, A.; KUCUK, H.; YAPAR, Z. A new model for single-layer drying. Drying Technology, v.20, n.7, p.1503-1513, 2002.

PAGE, G. E. Factors influencing the maximum rates of air drying shelled corn in thin layers. Purdue University, Ann Arbor, 1949.

PURKAYASTHA, M. D.; NATH, A.; DEKA, B. C.; MAHANTA, C. L. Thin layer drying of tomato slices. Journal of Food Science and Technology, v.50, n.4, p.642653, 2013.

SCHER, C. F.; DE OLIVEIRA RIOS, A.; NOREÑA, C. P. Z. Hot air drying of yacon (Smallanthus sonchifolius) and its effect on sugar concentrations. International Journal of Food Science \& Technology, v.44, n.11, p.2169-2175, 2009.

SHI, Q.; ZHENG, Y.; ZHAO, Y. Mathematical modeling on thin-layer heat pump drying of yacon (Smallanthus sonchifolius) slices. Energy Conversion and Management, v.71, p.208-216, 2013.

ZHU, A.; SHEN, X. The model and mass transfer characteristics of convection drying of peach slices. International Journal of Heat and Mass Transfer, v.72, p.345-351, 2014.

VAZ-TOSTES, M. D. G.; VIANA, M. L.; GRANCIERI, M.; LUZ, T. C. D. S.; PAULA, H. D.; PEDROSA, R. G.; COSTA, N. M. B. Yacon effects in immune response and nutritional status of iron and zinc in preschool 
children. Nutrition, v.30, n.6, p.666-672, 2014.

VEGA-GÁLVEZ, A.; AH-HEN, K.; CHACANA, M.; VERGARA, J.; MARTÍNEZ-MONZÓ, J.; GARCÍASEGOVIA, P.; LEMUS-MONDACA, R.; DI SCALA, K. Effect of temperature and air velocity on drying kinetics, antioxidant capacity, total phenolic content, colour, texture and microstructure of apple (var. Granny Smith) slices. Food Chemistry, v.132, n.1, p.51-59, 2012.
VILHENA, S. M. C.; CÂMARA, F. L. A.; KAKIHARA, S. T. O cultivo de yacon no Brasil. Horticultura Brasileira, v.18, n.1, p. 5-8, 2000.

\section{AGRADECIMENTOS}

Ao Conselho Nacional de Pesquisa e Desenvolvimento Tecnológico - CNPq, Coordenação de Aperfeiçoamento de Pessoal de Nível Superior-CAPES e Fundação de Amparo à Pesquisa do Estado de Minas Gerais - FAPEMIG, pelo apoio à pesquisa. 